\title{
Correlação entre a Espessura do Músculo Adutor do Polegar e 0 Estado Nutricional
}

doi: https://doi.org/10.32635/2176-9745.RBC.2020v66n4.1044

\author{
Correlation Between the Adductor Pollicis Muscle Thickness and the Nutritional Status \\ Correlación entre el Espesor del Músculo Adductor Pollicis y el Estado Nutricional
}

Camila Weschenfelder'; Sabrina Côrrea Salgueiro ${ }^{2}$

RESUMO

Introdução: A desnutrição é altamente prevalente na população oncológica e aumenta a morbidade e mortalidade nos estágios avançados da doença. A espessura do músculo adutor do polegar (EMAP) parece ser uma variável importante para avaliar o compartimento muscular. Objetivo: Estabelecer um ponto de corte da EMAP para desnutrição entre pacientes oncológicos hospitalizados. Método: Estudo transversal, entre 100 pacientes oncológicos internados em um hospital geral de Porto Alegre - RS, com idade $\geq 20$ anos, admitidos nas enfermarias e avaliados nas primeiras 72 horas de admissão hospitalar. Foi realizada a avaliação subjetiva global (ASG), mensurados peso, altura, circunferência de braço $(\mathrm{CB})$, prega cutânea triciptal (PCT), circunferência da panturrilha (CP) e a EMAP da mão dominante (EMAPD) e não dominante (EMAPND); calculada a circunferência muscular do braço (CMB) e o índice de massa corporal (IMC). Resultados: Conforme a ASG, 31\% e 33\% eram, respectivamente, moderadamente e gravemente desnutridos. Pacientes desnutridos apresentaram significativamente menores valores de IMC, CB, PCT, CMB, CP, EMAPD e EMAPND, bem como maior tempo de internação e óbito. O melhor ponto de corte da EMAPD para o desfecho desnutrição foi 13,2 mm (sensibilidade 65\% e especificidade 75\%) e, para EMAPND, foi 13,3 mm (sensibilidade de 65\% e especificidade 77\%). Conclusáo: O melhor ponto de corte da EMAPD para o desfecho desnutrição, proposto neste estudo, foi 13,2 mm e, para EMAPND, foi 13,3 mm. No entanto, mais estudos são necessários para confirmar estes achados.

Palavras-chave: Desnutrição; Avaliação Nutricional; Estado Nutricional; Antropometria; Neoplasias.

\section{ABSTRACT}

Introduction: Malnutrition is highly prevalent in the oncologic population and is the major cause of morbidity and mortality in the advanced stages of the disease. The adductor pollicis muscle thickness (APMT) seems to be an important variable to assess muscle compartment. Objective: To establish cutoff point for malnutrition from APMT among hospitalized oncologic patients. Method: Cross-sectional study with 100 oncologic patients hospitalized in a general hospital in Porto Alegre - RS, aged $\geq 20$ years, admitted at the outpatient and evaluated within the first 72 hours of hospital admission. Subjective Global Assessment (SGA) was performed; weight, height, arm circumference (AC), tricipital skinfold (TSF), calf circumference (CC), APMT of the dominant (APMTDH) and non-dominant hand (APMTNDH) were measured; arm muscle circumference (AMC) and body mass index (BMI) were calculated. Results: According to the SGA, 31\% of the sample was moderately malnourished and $33 \%$, severely malnourished. Malnourished patients had significantly lower values of BMI, AC, TSF, CC, APMTDH and APMTNDH, as well as, longer hospital stay and death. The best cutoff point for APMTDH for the malnutrition outcome was 13.2 $\mathrm{mm}$, (sensitivity of $65 \%$ and specificity of $75 \%$ ) and for APMTNDH, 13.3 $\mathrm{mm}$, with a sensitivity of $65 \%$ and specificity of $77 \%$. Conclusion: The best cutoff point proposed in this study for APMTDH for the outcome malnutrition was $13.2 \mathrm{~mm}$ and $13.3 \mathrm{~mm}$ for APMTNDH. However, further studies are needed to confirm our findings.

Key words: Malnutrition; Nutritional Assessment; Nutritional Status; Anthropometry; Neoplasms.

\section{RESUMEN}

Introducción: La desnutrición es altamente prevalente en la población con cáncer y aumenta la morbilidad y la mortalidad en las etapas avanzadas de la enfermedad. El espesor del músculo adductor pollicis (EMAP) parece ser una variable importante para evaluar el compartimento muscular. Objetivo: Establecer un punto de corte EMAP para la desnutrición en pacientes con cáncer hospitalizados. Método: Estudio transversal entre 100 pacientes con cáncer ingresados en un hospital general en Porto Alegre - RS, edad $\geq 20$ años y evaluados dentro de las primeras 72 horas de ingreso hospitalario. Se realizó una evaluación global subjetiva (EGS), se midió el peso, la altura, la circunferencia del brazo (CB), el pliegue cutáneo tricipital (PCT), la circunferencia de la pantorrilla (CP) y la EMAP de las manos dominantes (EMAPD) y no dominantes (EMAPND). Se calcularon la circunferencia muscular del brazo (CMB) y el índice de masa corporal (IMC). Resultados: Según la EGS, el 31\% y el 33\% estaban desnutridos moderada y severamente, respectivamente. Los pacientes desnutridos tuvieron valores significativamente más bajos de IMC, CB, PCT, CMB, CP, EMAPD y EMAPND, así como una mayor estadía hospitalaria y muerte. El mejor punto de corte para EMAPD para el resultado de desnutrición fue 13.2 $\mathrm{mm}$ (sensibilidad 65\% y especificidad 75\%), y para EMAPND fue 13.3 $\mathrm{mm}$ (sensibilidad $65 \%$ y especificidad $77 \%$ ). Conclusión: El mejor punto de corte para EMAPD para el resultado de desnutrición fue de $13.2 \mathrm{~mm}$ y para EMAPND fue de $13.3 \mathrm{~mm}$. Sin embargo, se necesitan más estudios para confirmar nuestros hallazgos.

Palabras clave: Desnutrición; Evaluación Nutricional; Estado Nutricional; Antropometría; Neoplasias.

${ }^{1}$ Departamento de Nutrição do Hospital Ernesto Dornelles. Programa de Pós-Graduação em Ciências de Saúde. Instituto de Cardiologia do Rio Grande do Sul. Porto Alegre (RS), Brasil. Orcid iD: https://orcid.org/0000-0002-9470-2569

2 Departamento de Nutrição do Hospital Ernesto Dornelles. Porto Alegre (RS), Brasil. Orcid iD: https://orcid.org/0000-0002-7071-3423

Endereço para correspondência: Camila Weschenfelder. Hospital Ernesto Dornelles. Avenida Ipiranga, 1801 - Praia de Belas. Porto Alegre (RS), Brasil. CEP 90160-093.

E-mail: camilawesche@gmail.com 


\section{INTRODUÇÃO}

A desnutrição é altamente prevalente na população oncológica e aumenta a morbidade e mortalidade nos estágios avançados da doença ${ }^{1,2}$; afeta negativamente a tolerância ao tratamento do câncer, a sobrevida dos pacientes, o tempo de internação, aumentando consequentemente os custos hospitalares ${ }^{2-4}$.

A caquexia associada ao câncer é caracterizada pela perda involuntária e progressiva do peso corporal e da massa muscular, sendo resultado da ação de fatores derivados do hospedeiro e do tumor, incluindo citocinas, que resultam em resposta inflamatória sistêmica 5 . Essa síndrome complexa e multifatorial é associada a anormalidades metabólicas, anorexia, saciedade precoce, edema, fadiga, função imune prejudicada e declínio da concentração mental ${ }^{6}$. Estima-se que cerca de $50 \%$ a $80 \%$ dos pacientes oncológicos apresentem caquexia e que esta seja responsável por $20 \%$ dos óbitos ${ }^{7}$.

Clinicamente, pacientes oncológicos apresentam sarcopenia, que parece ser a complicação mais deletéria, cuja prevalência varia entre $20 \%$ e $70 \%$, dependendo do tipo do tumor ${ }^{7}$. O risco de mortalidade está bem documentado nos pacientes sarcopênicos, já que a perda de massa muscular é associada ao aumento da resposta catabólica, à maior toxicidade do tratamento, ao maior risco de complicaçôes, à pior evoluçâo clínica e consequentemente à menor sobrevivência, mesmo na presença da obesidade, a qual soma a essa condição os riscos relacionados ao excesso de peso ${ }^{8}$.

Existem diversos métodos para avaliação do paciente hospitalizado. A avaliação subjetiva global (ASG) ${ }^{9}$ vem sendo utilizada em diversas condições clínicas. Pode ser considerada um marcador do estado de saúde, sendo o diagnóstico da desnutrição grave um indicador de gravidade da doença, e náo apenas um índice do déficit de nutrientes. No entanto, a Sociedade Europeia de Nutrição Clínica e Metabolismo (do inglês, European Society for Clinical Nutrition and Metabolism - ESPEN) ${ }^{10}$ recomenda a expansão das práticas de avaliaçáo relacionadas à nutrição, como a inclusão de medidas de anorexia, composição corporal, biomarcadores inflamatórios, função física, entre outros, em pacientes oncológicos.

Entre as medidas antropométricas, a avaliação da espessura do músculo adutor do polegar (EMAP) parece ser uma variável importante para avaliar o compartimento muscular, pois é considerada uma medida objetiva, rápida e de baixo custo, além de náo invasiva ${ }^{11-13}$ e ser útil para detectar alteraçôes precoces relacionadas com desnutriçáa ${ }^{12}$. Valores de referência e pontos de corte para desnutrição em relação à EMAP na população saudável já foram publicados ${ }^{14}$. No entanto, parece haver necessidade de se estabelecer a confiabilidade dos valores referentes ao ponto de corte da EMAP antes que ela possa ser usada como um componente da triagem nutricional em ambiente hospitalar ${ }^{15}$. Por fim, estudos que avaliaram a EMAP em ambiente hospitalar se concentram principalmente na populaçấo cirúrgica ${ }^{16-19}$, sendo escassa a literatura referente ao uso dessa técnica entre pacientes oncológicos ${ }^{20,21}$.

Uma vez que pacientes oncológicos possuem elevada prevalência de desnutrição e comprometimento da massa muscular, medidas que auxiliem na identificação dessas duas condiçôes são necessárias. Além disso, poucos estudos investigaram o valor dessa técnica como preditora do estado nutricional e de defechos clínicos nos pacientes oncológicos; por isso, o objetivo deste estudo foi estabelecer um ponto de corte da EMAP de ambas as mãos para desnutriçãa, considerando o diagnóstico da ASG como padrão-ouro entre pacientes oncológicos hospitalizados.

\section{MÉTODO}

Trata-se de um estudo transversal realizado em um hospital geral na cidade de Porto Alegre - RS, no qual a amostra foi definida por conveniência. Foram critérios de inclusão: pacientes oncológicos admitidos nas enfermarias, que pudessem ser avaliados nas primeiras 72 horas de admissão hospitalar, com idade $\geq 20$ anos e que consentiram sua participação por meio da assinatura do Termo de Consentimento Livre e Esclarecido (TCLE). Foram critérios de exclusão: instabilidade hemodinâmica, edema em membro superior e amputação de membro superior unilateral ou bilateral.

$\mathrm{Na}$ avaliação clínica, foi realizada a ASG ${ }^{9}$, que considera alteraçôes ponderais, na dieta e presença de sintomas, além do exame físico e da demanda metabólica da patologia de base para classificaçáo do estado nutricional. Neste estudo, utilizou-se a ASG, que classifica os pacientes em categorias A/B/C: (A) bem nutrido; (B) moderadamente desnutrido; ou (C) gravemente desnutrido. Para fins de análise, os pacientes com algum grau de desnutrição foram agrupados e o diagnóstico nutricional da ASG foi utilizado como padrão-ouro para estabelecer o ponto de corte da medida EMAP para desnutrição.

$\mathrm{O}$ peso dos indivíduos foi aferido em quilogramas (kg), por meio de balança digital Balmak; a altura, em centímetros, foi aferida por intermédio de estadiômetro e, a partir desses dois indicadores, foi calculado o índice de massa corporal (IMC), com ponto de corte $\leq 18,5 \mathrm{~kg} / \mathrm{m}^{2}$ para desnutrição de adultos até $60 \operatorname{anos}^{22} \mathrm{e} \leq 22 \mathrm{~kg} / \mathrm{m}^{2}$ para adultos a partir de 60 anos $^{23}$. Para os pacientes acamados, a estatura foi estimada pela altura do joelho e a estimativa 
de peso corporal, por meio da equação de Chumlea et al. ${ }^{24}$, para ambos os gêneros e ciclo da vida.

A circunferência do braço $(\mathrm{CB})$ e da panturrilha $(\mathrm{CP})$ foram aferidas em $\mathrm{cm}$, sendo mensuradas utilizando fita métrica inelástica, com precisão de $1 \mathrm{~mm}$, segundo as técnicas descritas em Lohman et al. ${ }^{25}$. Para CP, valores abaixo de $31 \mathrm{~cm}$ foram considerados indicativos de redução de massa muscular ${ }^{26}$. A prega cutânea triciptal (PCT) foi aferida com o plicômetro da marca Cescorf . A circunferência muscular do braço $(\mathrm{CMB})$ foi obtida a partir dos valores de CB e PCT, por meio da equação: $\mathrm{CMB}(\mathrm{cm})=\mathrm{CB}(\mathrm{cm})-\pi \mathrm{x}[\mathrm{PCT}(\mathrm{mm} / 10)]$. Para a classificação da $\mathrm{CMB}$, foram utilizados os valores em percentil propostos por Frisancho ${ }^{26}$.

A medida da EMAP foi realizada com o paciente sentado, o braço flexionado a aproximadamente $90^{\circ}$, o antebraço e a mão apoiados sobre o joelho. Os pacientes foram orientados a ficar com a mão relaxada. Foi utilizado o plicômetro da marca Cescorf ${ }^{ø}$ com pressão contínua de $10 \mathrm{~g} / \mathrm{mm}^{2}$ para pinçar o músculo adutor no vértice de um triângulo imaginário, formado pela extensão do polegar e indicador ${ }^{27}$. O procedimento foi realizado na mão não dominante (EMAPND) e na mão dominante (EMAPD), em triplicata, sendo utilizada a média como medida da EMAP. Para classificaçáo dos valores obtidos, foram definidos pontos de corte para ambas as mãos. A medida da EMAP foi realizada em todos os pacientes pelo mesmo nutricionista, que recebeu treinamento com profissionais de referência quanto à técnica necessária para avaliação da EMAP.

O programa Statistical Package for the Social Sciences (SPSS), versão 17.0 para Windows, foi utilizado para todas as análises estatísticas. As variáveis contínuas foram descritas em média e desvio-padrão e as categóricas como números absolutos e percentuais. Testes t de Student ou Wilcoxon Mann-Whitney, e teste exato de Fisher foram utilizados para comparaçôes. Para a seleção dos pontos de coorte da EMAP, foi adotada a análise a partir da técnica das curvas Receiver Operating Characteristic (ROC). A curva ROC foi gerada pela plotagem da sensibilidade no eixo y em funçáo de 1 - especificidade - no eixo x. A significância estatística de cada análise foi verificada pela área sob a curva ROC e pelo intervalo de confiança de $95 \%$ (IC 95\%). Foram apresentados os respectivos IC 95\% e os valores de $\mathrm{p}<0,05$ foram considerados significativos.

\section{RESULTADOS}

Entre novembro de 2016 e março de 2017, foram incluídos 100 pacientes, sendo 38 homens (38\%), 62 mulheres (62\%). A média de idade foi de $66,1 \pm 15,9$ anos e o IMC médio de $24,7 \pm 5,1 \mathrm{~kg} / \mathrm{m}^{2}$. Os pacientes foram

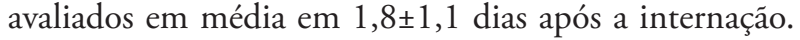
A mediana do tempo de internação foi de 10 dias (IC 95\% 7,9-12,1) e foram registrados 13 óbitos. De acordo com a classificação da ASG, 36\% estavam bem nutridos, $31 \%$ moderadamente desnutridos e $33 \%$ gravemente desnutridos.

A média da EMAPD foi 14 4 4,64 mm e da EMAPND foi $13,4 \pm 4,1 \mathrm{~mm}$. A Tabela 1 apresenta as características da amostra de acordo com o estado nutricional. Pacientes classificados como desnutridos apresentaram significativamente menores valores de IMC, CB, PCT, $\mathrm{CMB}, \mathrm{CP}$, EMAPND e EMAPD, bem como maior tempo de internação e óbito.

Tabela 1. Características dos participantes de acordo com a avaliação subjetiva global [média \pm desvio-padrão, mediana (IQR) ou $n(\%)]$

\begin{tabular}{lclc}
\hline & $\begin{array}{c}\text { Nutridos } \\
\text { (ASG=A) }\end{array}$ & $\begin{array}{l}\text { Desnutridos } \\
(\mathbf{A S G}=\mathbf{B} \text { e C) }\end{array}$ & Valor $\mathbf{p}$ \\
\hline $\begin{array}{l}\text { Idade (anos) } \\
\text { Gênero }\end{array}$ & $63,6 \pm 15,8$ & $67,5 \pm 15,9$ & $0,23^{*}$ \\
$\quad$ Homens & $15(38,5)$ & $23(60,5)$ & $0,67^{\dagger}$ \\
IMC (Kg/m²) & $28,03 \pm 4$ & $22,84 \pm 4,8$ & $<0,01^{*}$ \\
$\mathrm{CB}(\mathrm{cm})$ & $30,9 \pm 3,1$ & $24,9 \pm 4,8$ & $<0,01^{*}$ \\
$\mathrm{PCT}(\mathrm{mm})$ & $18,9 \pm 7,8$ & $13,6 \pm 6$ & $<0,01^{*}$ \\
$\mathrm{CMB}(\mathrm{cm})$ & $25 \pm 3,9$ & $21,5 \pm 3,5$ & $<0,01^{*}$ \\
$\mathrm{CP}(\mathrm{cm})$ & $36,4 \pm 5,9$ & $31,8 \pm 6,2$ & $<0,01^{*}$ \\
EMAPD & $16,6 \pm 5,4$ & $12,6 \pm 3,5$ & $<0,01^{*}$ \\
EMAPND & $15,8 \pm 3,7$ & $12,1 \pm 3,7$ & $<0,01^{*}$ \\
Tempo & & & \\
internação & $5(3-8)$ & $8(6-13)$ & $<0,01^{\ddagger}$ \\
(dias) & & & \\
Desfecho & & & $0,03^{\dagger}$ \\
$\quad$ Óbito & $1(7,7)$ & $12(92,3)$ & \\
\hline
\end{tabular}

Legendas: IMC: índice de massa corporal; CB: circunferência do braço; PCT: prega cutânea tricipital; $\mathrm{CMB}$ : circunferência muscular do braço; $\mathrm{CP}$ : circunferência da panturrilha; EMAPD: espessura do músculo adutor do polegar da mão dominante; EMAPND: espessura do músculo adutor do polegar da mão não dominante. *Teste t de Student; †teste exato de Fisher; łteste de WilcoxonMann-Whitney.

No total, 36 indivíduos apresentaram o diagnóstico prévio de hipertensão, 18 indivíduos de diabetes mellitus e sete de doença pulmonar obstrutiva crônica. A Figura 1 contém a frequência das neoplasias. Os cânceres mais frequentes foram o de intestino e o de mama, sendo 19\% dos pacientes portadores de neoplasia metastática.

$\mathrm{Na}$ Figura 2, estáo representadas a curva ROC da EMAP de ambas as máos e referidas as respectivas áreas sob a curva para essa populaçáo. $\mathrm{O}$ melhor ponto de 


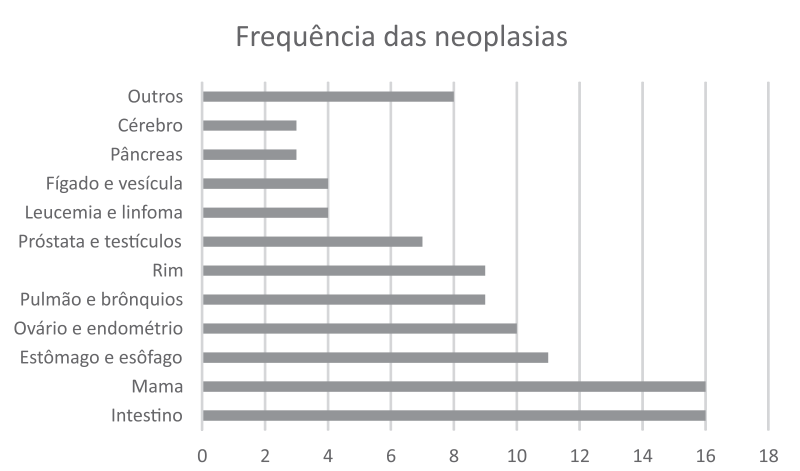

Figura 1. Frequência das neoplasias na população avaliada

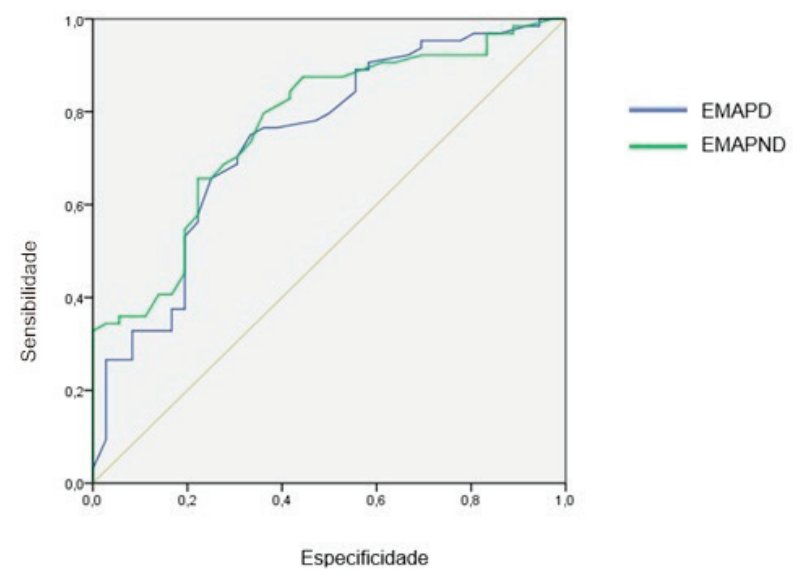

Figura 2. Relação da EMAP com a desnutrição

Legendas: EMAPD: espessura do músculo adutor do polegar da mão dominante (área sob a curva: 0,74 [IC 95\% 0,64-0,84]); EMAPND: espessura do músculo adutor do polegar da mão náo dominante (área sob a curva: 0,77 [IC 95\% $0,68-0,86])$

corte da EMAPD para o desfecho desnutriçáo foi 13,2 $\mathrm{mm}$, com sensibilidade de $65 \%$ e especificidade de $75 \%$ e, para EMAPND, foi 13,3 mm, com sensibilidade de 65\% e especificidade de $77 \%$.

Entre 51 indivíduos considerados desnutridos pela EMAPD e EMAPND, foram classificados como nutridos pela ASG, respectivamente, nove e oito. A Tabela 2 apresenta as variáveis antropométricas de acordo com o ponto de corte da EMAP para desnutrição. Para ambas as máos, os valores de IMC, $\mathrm{CB}, \mathrm{CMB}$ e $\mathrm{CP}$ foram significativamente inferiores nos pacientes desnutridos em comparação aos nutridos. A EMAPD apresentou correlação com CP, IMC, CB e CMB (todos, $\mathrm{p}<0,01$ ) e não foi correlacionada estatisticamente com a PCT $(\mathrm{p}=0,07)$. A EMAPND apresentou correlaçáo com CP, IMC, CB (todos, $\mathrm{p}<0,01)$, PCT $(\mathrm{p}=0,01)$ e CMB $(\mathrm{p}=0,02)$.

\section{DISCUSSÃO}

Os resultados deste estudo demonstram que os pontos de corte estabelecidos para o diagnóstico de desnutrição pela EMAP de ambas as mãos tiveram valores de sensibilidade e especificidade relativamente baixos (respectivamente, EMAPD 65\% e 75\%; EMAPND $65 \%$ e $77 \%$ ). Por se tratar de ponto de corte inicialmente pensado para realizar o diagnóstico nutricional, valores altos de especificidade seriam esperados. Diferentemente, Gonzalez et al. ${ }^{16}$ compararam os resultados da EMAP com a ASG entre pacientes cirúrgicos e observaram que a EMAP apresentou baixa sensibilidade (dominante: $34,9 \%$, não dominante: $37,7 \%$ ), mas alta especificidade (dominante: $98,7 \%$, não dominante: $97,8 \%$ ) para identificar desnutriçấo.

Outro estudo na população cirúrgica ${ }^{12}$ observou pontos de corte para desnutrição conforme a EMAP das mãos direita e esquerda, respectivamente, de 13,4 $\mathrm{mm}$ e $13,1 \mathrm{~mm}$, sensibilidade de $72,37 \%$ e $77,33 \%$, e especificidade de $100 \%$ para ambas as mãos. Neste sentido, considerando estes achados, os da população cirúrgia e os dados de revisão sistemática publicada previamente ${ }^{15}$, acredita-se que outros estudos na população oncológica sejam necessários até que se possa estabelecer um ponto de corte para essa população.

Tabela 2. Variáveis antropométricas de acordo com o ponto de corte da EMAP para desnutrição [média \pm desvio-padrão]

\begin{tabular}{|c|c|c|c|c|c|c|}
\hline & \multicolumn{3}{|c|}{ EMAPD } & \multicolumn{3}{|c|}{ EMAPND } \\
\hline & $\begin{array}{c}\text { Eutrófico } \\
(n=49)\end{array}$ & $\begin{array}{l}\text { Desnutrido } \\
\qquad(n=51)\end{array}$ & Valor p* & $\begin{array}{c}\text { Eutrófico } \\
(n=51)\end{array}$ & $\begin{array}{c}\text { Desnutrido } \\
(n=49)\end{array}$ & Valor $\mathbf{p}^{*}$ \\
\hline $\mathrm{IMC}\left(\mathrm{Kg} / \mathrm{m}^{2}\right)$ & $27 \pm 4,3$ & $22,5 \pm 4,9$ & $<0,01$ & $26,8 \pm 4,4$ & $22,5 \pm 4,9$ & $<0,01$ \\
\hline $\mathrm{CB}(\mathrm{cm})$ & $29,5 \pm 3,4$ & $24,8 \pm 5,3$ & $<0,01$ & $29,4 \pm 3,5$ & $24,6 \pm 5,3$ & $<0,01$ \\
\hline PCT (mm) & $17,1 \pm 8$ & $14,2 \pm 6,1$ & 0,09 & $17,1 \pm 7,8$ & $14 \pm 6,1$ & 0,07 \\
\hline $\mathrm{CMB}(\mathrm{cm})$ & $24 \pm 3,7$ & $21,9 \pm 4,1$ & 0,02 & $23,8 \pm 3,7$ & $21,9 \pm 4,2$ & 0,05 \\
\hline $\mathrm{CP}(\mathrm{cm})$ & $36 \pm 5,3$ & $30,9 \pm 6,4$ & $<0,01$ & $35,9 \pm 5,3$ & $30,8 \pm 6,5$ & $<0,01$ \\
\hline
\end{tabular}

Legendas: IMC: índice de massa corporal; CB: circunferência do braço; PCT: prega cutânea tricipital; CMB: circunferência muscular do braço; CP: circunferência da panturrilha; EMAPD: espessura do músculo adutor do polegar da mão dominante; EMAPND: espessura do músculo adutor do polegar da mão não dominante. *Teste t de Student. 
$\mathrm{Na}$ população saudável, as médias de EMAP entre homens e mulheres são, respectivamente, $26,1 \pm 4,4 \mathrm{~mm}$ e 19,8 $\pm 3,3 \mathrm{~mm}$ para EMAPD; $25,1 \pm 4,4 \mathrm{~mm}$ e $18,7 \pm 3,1$ $\mathrm{mm}$ para EMAPND ${ }^{28}$. No entanto, os pontos de corte de EMAP para definir risco nutricional e/ou desnutrição parecem variar amplamente de acordo com a população estudada. Entre cardipatas internados em unidade de terapia intensiva, o ponto de corte da EMAPD associado ao desfecho desnutriçấo foi $<6,5 \mathrm{~mm}$ (área sob a curva 0,82; IC $95 \%$ de 0,73 a 0,91$)^{29}$.

Neste estudo, a EMAP apresentou similaridade com as demais variáveis antropométricas utilizadas para avaliação do compartimento muscular e que são relevantes ao se estabelecer um diagnóstico nutricional em pacientes oncológicos hospitalizados. Os pacientes classificados como desnutridos pela ASG apresentaram significativamente maior tempo de internação e óbito, e menores valores de IMC, CB, PCT, CMB, CP, EMAPD e EMAPND. Além disso, verifica-se que o ponto de corte para EMAP de ambas as mãos se associou às demais variáveis antropométricas, com exceção da PCT.

Em relação à associação entre EMAP, ASG e as demais variáveis antropométricas, alguns estudos similares foram conduzidos na população oncológica. Estudo de Silva et al. ${ }^{30}$ avaliou o estado nutricional de 43 pacientes oncológicos, considerando EMAP, IMC, PCT, CP, entre outros. Houve pequena concordância entre os parâmetros utilizados, como a EMAP e ASG, mas foram obtidas diferenças significativas entre os valores de CB e EMAP, indicando que esses parâmetros podem ser úteis na identificação de pacientes nutridos e desnutridos, desde que definidos valores de ponto de corte.

De forma semelhante a estes achados, Poziomyck et al. ${ }^{21}$ estudaram 74 adultos e idosos, submetidos à ressecção de tumores do trato gastrointestinal, com o objetivo de avaliar qual seria o método de avaliaçáo nutricional mais sensível nesse grupo. Utilizaram-se ASG, EMAP, IMC, CB, $\mathrm{CMB}$, percentual de perda de peso e PCT, além de exames bioquímicos. Os resultados revelaram que a $\operatorname{EMAP}(<8,8 \mathrm{~mm})$ e a ASG ( $\geq \mathrm{B}$ ) foram confiáveis na previsão de mortalidade e podem ser utilizadas na prática clínica $(\mathrm{p}<0,01)$.

Entre pacientes cirúrgicos, Melo et al. ${ }^{18}$ realizaram um estudo para estimar a prevalência de desnutriçáo pela EMAP em 151 pacientes cirúrgicos eletivos. Foram utilizadas medidas antropométricas como CB, PCT e IMC, percentual de perda de peso e medida da EMAP em ambas as mãos. Os autores observaram alta prevalência de desnutrição, além de associação significativa entre o diagnóstico nutricional segundo EMAP e as medidas de $\mathrm{CB}, \mathrm{PCT}$ e IMC, demonstrando que o músculo adutor parece ser bom método para diagnosticar depleçâo muscular e desnutrição nessa população.
Estudo de Bragagnolo et al. ${ }^{17}$ avaliou 124 pacientes submetidos à cirurgia de grande porte do trato gastrointestinal e concluiu que a EMAP pode ser utilizada como preditora de mortalidade ( $\mathrm{RR}=1,26$; IC 95\%: 1,03-1,55; $p=0,02$ ) e tende a ser preditora de complicaçóes, além de ser uma importante ferramenta para avaliação do estado nutricional em pacientes cirúrgicos. Este estudo ainda avaliou a associação da EMAP com a ASG, que apresentaram correlaçáo significativa entre elas, embora a ASG não tenha permanecido no modelo final pela análise de regressão. A CMB foi a variável que mais influenciou na medida da EMAP nessa população após os ajustes.

Este estudo possui limitaçóes. Por se tratar de um estudo exploratório com delineamento de pesquisa transversal, pode ter havido interpretação de causalidade reversa. $\mathrm{O}$ espectro da população avaliada (pacientes oncológicos atendidos em um hospital geral, com altas prevalências das formas mais graves da doença) pode ter limitado a inferência dos resultados. A amostra (definida por conveniência) pode não ter sido suficiente para análise dessas variáveis.

\section{CONCLUSÃO}

O melhor ponto de corte da EMAPD para o desfecho desnutrição proposto neste estudo foi 13,2 $\mathrm{mm}$ e, para EMAPND, 13,3mm. A utilização de indicadores de desnutrição para avaliação do risco nutricional entre a populaçấo em geral já está estabelecida e, entre pacientes oncológicos, ainda há necessidade de um número maior de estudos para a determinação dos indicadores antropométricos mais robustos, no que se refere a associaçóes entre o perfil nutricional alterado e a predição de risco para desfechos.

\section{CONTRIBUIÇÕES}

Camila Weschenfelder contribuiu substancialmente na concepção e planejamento do estudo, na análise e interpretaçáo dos dados, assim como na redaçáo e revisão crítica. Sabrina Côrrea Salgueiro contribuiu na obtenção, análise e interpretação dos dados, assim como na redação e revisão crítica. Ambas as autoras aprovaram a versão final a ser publicada.

\section{DECLARAÇÃO DE CONFLITO DE INTERESSES}

Nada a declarar.

\section{FONTES DE FINANCIAMENTO}

Não há. 


\section{REFERÊNCIAS}

1. Stratton, RJ, Green CJ, Elia M, editors. Disease-related malnutrition: an evidence-based approach to treatment. Wallingford (UK): CABI Publishing; 2003. Prevalence of disease-related malnutrition; p. 35-92.

2. Arends J, Bachmann P, Baracos V, et al. ESPEN guidelines on nutrition in cancer patients. Clin Nutr. 2017;36(1):11-48. doi: https://doi.org/10.1016/j. clnu.2016.07.015

3. Tian J, Chen JS. Nutritional status and quality of life of the gastric cancer patients in Changle County of China. World J Gastroenterol. 2005;11(11):1582-6. doi: https:// doi.org/10.3748/wjg.v11.i11.1582

4. van Cutsem E, Arends J. The causes and consequences of cancer-associated malnutrition. Eur J Oncol Nurs. 2005;9(Suppl 2):S51-S63. doi: https://doi. org/10.1016/j.ejon.2005.09.007

5. Argilés JM, Busquets S, Stemmler B, et al. Cancer cachexia: understanding the molecular basis. Nat Rev Cancer. 2014;14:754-62. doi: https://doi.org/10.1038/ nrc3829

6. Tisdale MJ. Cancer anorexia and cachexia. Nutrition. 2001;17(5):438-42. doi: https://doi.org/10.1016/s08999007(01)00506-8

7. Ryan AM, Power DG, Daly L, et al. Cancer-associated malnutrition, cachexia and sarcopenia: the skeleton in the hospital closet 40 years later. Proc Nutr Soc, 2016;75(2):199-211. doi: https://doi.org/10.1017/ S002966511500419X

8. Miján de la Torre A. El músculo, elemento clave para la supervivencia en el enfermo neoplásico. Nutr Hosp. 2016;33(Supl 1):11-16.

9. Detsky AS, McLaughlin JR, Baker JP, et al. What is subjective global assessment of nutritional status? JPEN J Parenter Enteral Nutr. 1987;11(1):8-13. doi: https:// doi.org/10.1177/014860718701100108

10. Arends J, Baracos V, Bertz H, et al. ESPEN expert group recommendations for action against cancer-related malnutrition. Clin Nutr. 2017;36(5):1187-96. doi: https://doi.org/10.1016/j.clnu.2017.06.017

11. Pereira PML, Neves FS, Bastos MG, et al. Adductor pollicis muscle thickness for nutritional assessment: a systematic review. Rev Bras Enferm. 2018;71(6):30933102. doi: https://doi.org/10.1590/0034-7167-20170913

12. Bragagnolo R, Caporossi FS, Dock-Nascimento DB, et al. Espessura do músculo adutor do polegar: um método rápido e confiável na avaliação nutricional de pacientes cirúrgicos. Rev Col Bras Cir. 2009;36(5):371-6. doi: https://doi.org/10.1590/S0100-69912009000500003

13. Andrade PV, Lameu EB. Espessura do músculo adutor do polegar: um novo índice prognóstico em pacientes clínicos. Rev Bras Nutr Clin. 2007;22(1):28-35.
14. Gonzalez MC, Duarte RRP, Budziareck MB. Adductor pollicis muscle: reference values of its thickness in a healthy population. Clin Nutr. 2010;29(2):268-71. doi: https://doi.org/10.1016/j.clnu.2009.08.012

15. Lew CCH, Ong F, Miller M. Validity of the adductor pollicis muscle as a component of nutritional screening in the hospital setting: a systematic review. Clin Nutr ESPEN. 2016;16:1-7. doi: https://doi.org/10.1016/j. clnesp.2016.08.005

16. Gonzalez MC, Pureza Duarte RR, Orlandi SP, et al. Adductor pollicis muscle: a study about its use as a nutritional parameter in surgical patients. Clin Nutr. 2015;34(5):1025-9. doi: https://doi.org/10.1016/j. clnu.2014.11.006

17. Bragagnolo R, Caporossi FS, Dock-Nascimento D, et al. Handgrip strength and adductor pollicis muscle thickness as predictors of postoperative complications after major operations of the gastrointestinal tract. E Spen Eur E J Clin Nutr Metab. 2011;6:e21-e26. doi: https://doi. org/10.1016/j.eclnm.2010.11.001

18. Melo CYSV, Silva SA. Músculo adutor do polegar como preditor de desnutrição em pacientes cirúrgicos. ABCD, Arq Bras Cir Dig. 2014;27(1):13-7. doi: https://doi. org/10.1590/s0102-67202014000100004

19. Poziomyck AK, Corleta OC, Cavazzola LT, et al. Adductor pollicis muscle thickness and prediction of postoperative mortality in patients with stomach cancer. ABCD, Arq Bras Cir Dig. 2018;31(1):e1340. doi: https://doi.org/10.1590/0102-672020180001e1340

20. Valente KP, Almeida BL, Lazzarini TR, et al. Association of adductor pollicis muscle thickness and handgrip strength with nutritional status in cancer patients. PLoS One. 2019;14(8):e0220334. doi: https://doi. org/10.1371/journal.pone.0220334

21. Poziomyck AK, Weston AC, Lameu EB, et al. Preoperative nutritional assessment and prognosis in patients with foregut tumors. Nutr Cancer. 2012;64(8):1174-81. doi: https://doi.org/10.1080/01635581.2012.721157

22. Organização Mundial da Saúde (OMS). Obesidade: prevenindo e controlando a epidemia global. Sáo Paulo: Roca; 2004.

23. Organización Panamericana de la Salud, División de Promoción y Protección de la Salud (HPP). Encuesta multicéntrica salud bienestar y envejecimiento (SABE) en América Latina: informe preliminar [Internet]. In: XXXVI Reunion del Comite Asesor de Investigaciones en Salud; 2001 jul 9-11; Kingston, Jamaica. Washington (DC): OPAS; 2001. 19 p [acesso 2020 jun 21]. Disponível em: https://iris.paho.org/handle/10665.2/45890

24. Chumlea WC, Guo S, Roche AF, et al. Prediction of body weight for the nonambulatory elderly from anthropometry. J Am Diet Assoc, 1988;88(5):564-8.

25. Lohman TG, Roche AF, Martorell R, editors. Anthropometric standardization reference manual. Champaign: Human Kinetics; 1988. 
26. Frisancho AR. Anthropometric standards for the assessment of growth and nutritional status. Michigan: University of Michigan Press; 1990.

27. Lameu EB, Gerude MF, Corrêa RC, et al. Adductor policis muscle: a new anthropometric parameter. Rev Hosp Clin. 2004;59(2):57-62. doi: https://doi. org/10.1590/S0041-87812004000200002

28. Gonzalez MC, Duarte RRP, Budziareck MB. Adductor pollicis muscle: reference values of its thickness in a healthy population. Clin Nutr. 2010;29(2):268-71. doi: https://doi.org/10.1016/j.clnu.2009.08.012

29. Karst FP, Vieira RM, Barbiero S. Relationship between adductor pollicis muscle thickness and subjective global assessment in a cardiac intensive care unit. Rev Bras Ter Intensiva. 2015;27(4):369-75. doi: https://doi. org/10.5935/0103-507X.20150062

30. Silva JB, Maurício SF, Bering T, et al. The relationship between nutritional status and the Glasgow prognostic score in patients with cancer of the esophagus and stomach. Nutr Cancer. 2013;65(1):25-33. doi: https:// doi.org/10.1080/01635581.2013.741755

Recebido em 16/5/2020

Aprovado em 23/6/2020 